\title{
Gastrointestinal Stromal Tumors and Other Mesenchymal Lesions of the Gut
}

Joel K. Greenson, M.D.

Department of Pathology, University of Michigan Health System, Ann Arbor, Michigan

In the past 5 years, there has been a paradigm shift in our understanding of gastrointestinal stromal tumors (GISTs). Once thought to be smooth muscle tumors, these uncommon neoplasms are now thought to differentiate along the lines of interstitial cells of Cajal, the pacemaker cells of the gut. Along with this understanding comes an exciting new drug therapy (Gleevec) that for the first time offers real hope to patients with malignant stromal tumors. Overall, approximately $60-70 \%$ of stromal tumors are from the stomach, $20-30 \%$ are from the small intestine, and $<10 \%$ come from the esophagus, colon, rectum, omentum, and mesentery. Between 10 and $30 \%$ of GISTs are malignant. Stromal tumors should be studied in a site-specific fashion, as tumors from a given location in the gut have unique growth patterns and corresponding behaviors. Although the most important tool needed to diagnose a GIST is still a hematoxylin and eosinstained section, a confirmatory CD117 stain is recommended (and may be required for drug therapy). True smooth muscle tumors, inflammatory fibroid polyps, fibromatoses, schwannomas, inflammatory myofibroblastic tumors, and solitary fibrous tumors all enter into the differential diagnosis of GISTs. This article reviews the histologic features of these tumors in the context of recent molecular genetic and immunohistochemical advances.

KEY WORDS: c-Kit, CD117, Fibromatosis, Gastrointestinal stromal tumor (GIST), Inflammatory fibroid polyp, Leiomyoma, Leiomyosarcoma, Pathology, Prognosis, Schwannoma.

Mod Pathol 2003;16(4):366-375

Copyright $\odot 2003$ by The United States and Canadian Academy of Pathology, Inc.

VOL. 16, NO. 4, P. 366, 2003 Printed in the U.S.A.

Date of acceptance: January 8, 2003.

Address reprint requests to: Joel K. Greenson, M.D., Dept. of Pathology, Rm 2G332, Box 0054, 1500 E. Medical Center Drive, University of Michigan Health System, Ann Arbor, MI 48109-0054; fax: 734-763-4095; e-mail: facjkgmd@umich.edu.

DOI: 10.1097/01.MP.0000062860.60390.C7
Although a great many mesenchymal lesions may be found in the gastrointestinal (GI) tract, none are more vexing to the surgical pathologist than stromal tumors. In 1960, Martin and colleagues (1) published a series of six cases of "intramural myoid tumors" of the stomach and theorized that these unusual tumors were of smooth muscle origin. Two years later, Stout (2) introduced the term leiomyoblastoma to describe a group of bizarre smooth muscle tumors of the stomach. Since that time, a wide variety of names have been used to describe these spindle and epithelioid cell tumors that may arise anywhere along the GI tract as well as in the omentum and mesentery. In 1983, Mazur and Clark (3) first used the term stromal tumor to describe the lack of markers of differentiation in many of these lesions. Whereas some tumors were thought to differentiate along the lines of smooth muscle cells, others were interpreted as being neurogenic. There is no telling how many thousands of hours have been spent by researchers performing electron microscopy or countless immunostains in an attempt to find this holiest of grails-the cell of origin of gastrointestinal stromal tumors (GISTs). More than one good forest has been sacrificed in our quest, as paper after paper has been published describing the latest antibody staining pattern or ultrastructural analysis. To add to the confusion, true leiomyomas and schwannomas were frequently trapped within the all-encompassing term of stromal tumor. The finding that the majority of stromal tumors stained with CD34, a marker of myeloid progenitor cells, endothelial cells, and some mesenchymal lesions, helped separate GISTs from most true leiomyomas and schwannomas (which are CD34 negative; 4). CD34 positivity in GISTs ranges from 46 to $100 \%$, depending on the site of origin of the tumor $(4,5)$. The least CD34 positivity is seen in small-bowel GISTs (4). Although the discovery of CD34 staining in stromal tumors helped somewhat to further define these lesions, the fact that a fair number of GISTs were CD34 negative was problematic.

In the past 5 years, several seminal works involving the c-kit proto-oncogene have greatly expanded our understanding of stromal tumors. C-kit is a transmembrane tyrosine kinase receptor that be- 
longs to the same family of receptors as colonystimulating factor-1 (6). The ligand for c-kit is known as stem cell factor, and the gene coding for c-kit is on chromosome 4, near the gene for epidermal growth factor (6). Stem cell factor c-kit interaction is necessary for the proper development of melanocytes, erythrocytes, germ cells, mast cells, and the interstitial cells of Cajal (ICC; 7). Activating c-kit mutations have been identified in tumors affecting these cell lineages (8). Hirota and colleagues (7) were the first to describe the presence of such activating mutations in GISTs. Those investigators also showed that the majority (94\%) of GISTs stained positively with a polyclonal c-kit antibody, CD117 (7). They also showed that ICCs stain with antibodies to both CD34 and CD117 and postulated that GISTs may originate from ICCs (7). Kindblom and colleagues (9) found that $100 \%$ of their GISTs were CD117 positive and postulated that GISTs may arise from stem cells that differentiate toward ICCs. They proposed changing the name from stromal tumors to gastrointestinal pacemaker cell tumors, or GIPACT, to reflect this proposed origin. Both Hirota et al. (9) and Kindblom et al. (9) found that "true" leiomyomas and schwannomas had negative staining for CD117.

If these findings weren't enough to drive most stromal tumor mavens into fits of ecstasy, the discovery of a drug that successfully treats malignant GISTs by inhibiting the tyrosine kinase activity of c-kit is nothing short of miraculous. STI571, which is now known by its trade name Gleevec (Novartis, Basel, Switzerland), is a compound that was specifically designed to inhibit the Abl protein tyrosine kinase, which is present in $95 \%$ of patients with chronic myelogenous leukemia $(10,11)$. It now appears that this compound can inhibit several families of tyrosine kinases (including c-kit), and hence it is now being evaluated in the treatment of a variety of neoplasms. Clinical trials have found that this compound is very effective in treating malignant GISTs and that it causes minimal side effects (12). This is a major improvement for patients with metastatic or recurrent stromal tumors, as there has not been an effective therapy for these lesions. The only drawback to Gleevec is that the patients may need to take it for the rest of their lives, at a cost of about $\$ 2000 /$ month.

The development of an effective therapy for malignant GISTs has important implications, not only for patients but also for surgical pathologists. Predicting the biological behavior of GISTs is a difficult and somewhat controversial endeavor. Although some experts believe that benign stromal tumors exist, others believe that all lesions should be classified as being of at least low malignant potential. The problem with predicting prognosis in these lesions is due to several factors. GISTs are fairly uncommon tumors whose behaviors are somewhat different depending upon their sites of origin (stomach versus small bowel versus colon, etc.) and these tumors may recur or metastasize 20 years after primary resection. This combination of factors makes it very hard for a single institution to gather enough cases with adequate clinical follow-up to have the statistical power to adequately assess outcome. As a result, all studies of GISTs and prognosis have been retrospective, and many have mixed tumors from several sites within the gut to have enough tumors. Although none of us would consider combining adenocarcinomas from the esophagus, stomach, small bowel, and colon to study the prognosis of carcinomas, we seem to have no problem doing this with stromal tumors. The best evidence that stromal tumors from different sites behave differently comes from a study by Emory and co-workers (13) at the Armed Forces Institute of Pathology (AFIP). They found that in a multivariate analysis of $>1000$ stromal tumors of the gut, tumor location was an independent predictor of outcome. Overall, approximately $60-70 \%$ of stromal tumors are from the stomach, $20-30 \%$ are from the small intestine, and $<10 \%$ come from the esophagus, colon, rectum, omentum, and mesentery $(14,15)$. Between 10 and $30 \%$ of GISTs are malignant (14). The most common sites of recurrence and metastasis are the omentum/peritoneum and the liver. Most stromal tumor patients are middle-aged (50-60 years old), and except for rare cases of neurofibromatosis Type 1 and extremely rare families with germline c-kit mutations, risk factors for these tumors are unknown $(13,14)$.

\section{STOMACH}

Up to $80 \%$ of gastric stromal tumors are benign. The features that correlate with malignant behavior are a high mitotic rate, large size, and invasion of the mucosa (16-19). Gastric stromal tumors have a few characteristic growth patterns that are not seen elsewhere in the gut. One such pattern is that of a densely cellular spindle cell tumor with perinuclear vacuoles and occasional palisading (Fig. 1). In a study by Appelman and Helwig (18), only 1 of 49 such tumors metastasized. The single tumor that metastasized was quite large and had $>5$ mitoses per 50 high-power fields (HPFs). Malignant spindle cell gastric stromal tumors tend to lose these vacuoles (Fig. 2). Another curious pattern of growth in gastric stromal tumors is the epithelioid variant (Stout's leiomyoblastoma). These tumors are composed of plump or rounded cells, many of which show marked nuclear pleomorphism (Fig. 3). Ironically, tumors with such pleomorphism often have few if any mitoses and typically behave in a benign 


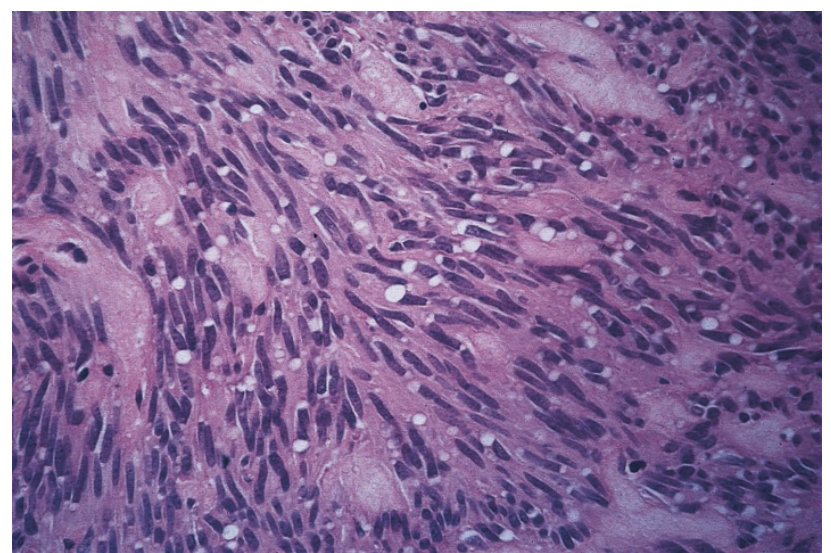

FIGURE 1. High-power photomicrograph of a benign spindle cell gastric stromal tumor showing characteristic nuclear palisading and perinuclear vacuoles.

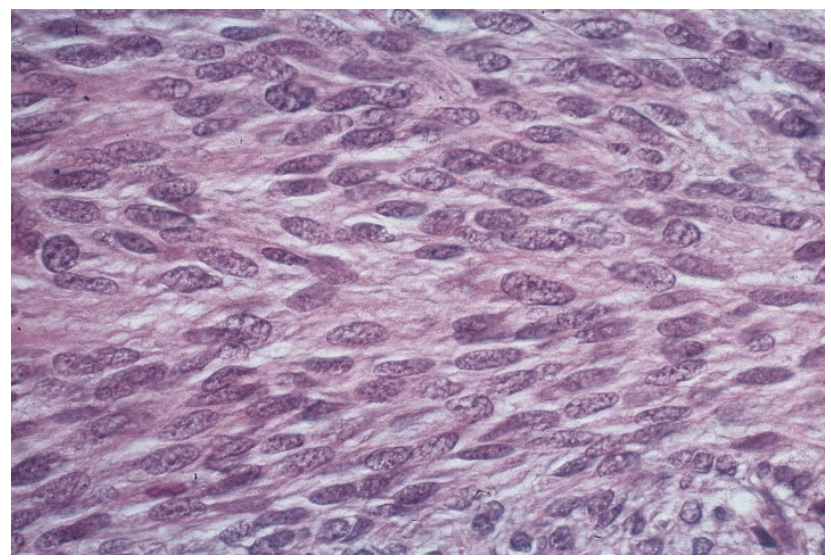

FIGURE 2. High-power photomicrograph of a malignant spindle cell gastric stromal tumor showing high cellularity with nuclear overlap and loss of perinuclear vacuoles (compare with Fig. 1).

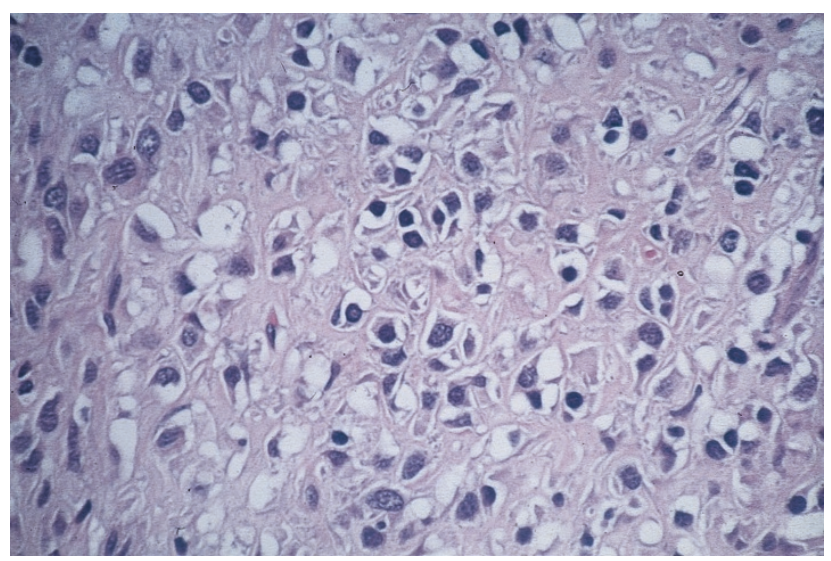

FIGURE 3. High-power photomicrograph of a benign epithelioid gastric stromal tumor showing nuclear pleomorphism without mitotic activity.

fashion (19). Malignant epithelioid stromal tumors of the stomach typically have smaller, more homogeneous cells (Fig. 4; 19). The presence of malignant epithelioid gastric stromal tumors in young women

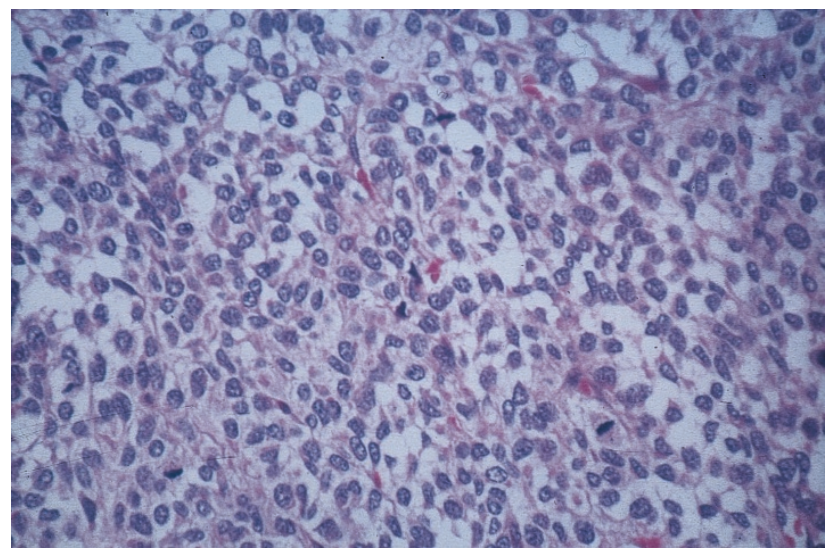

FIGURE 4. High-power photomicrograph of a malignant epithelioid gastric stromal tumor showing mitotic figures and a lack of nuclear pleomorphism (compare with Fig. 3).

is a marker of Carney's triad (20). These patients may have multiple malignant epithelioid gastric GISTs as well as paragangliomas and pulmonary chondromas (20). Despite having multiple malignant GISTs, these patients tend to have a better than expected prognosis.

The use of absolute cutoff values for size and mitoses in predicting behavior is somewhat controversial. In general, mitotic counts of $\geq 5$ per $50 \mathrm{HPFs}$ are considered malignant. Some studies use a size of $>5 \mathrm{~cm}$ as an indicator of malignancy, whereas others use $6 \mathrm{~cm}$. My own bias is that size alone is not a good predictor of malignancy. The problem, of course, is that not every tumor follows these rules: many histologically malignant-appearing lesions never metastasize and, rarely, benignappearing lesions do. This has led some experts to propose calling all GISTs potentially malignant.

\section{DUODENUM}

Duodenal stromal tumors, although not common, are most often found in the second part of the duodenum. Unlike the case with the stomach, about half of duodenal stromal tumors are malignant (21). Benign lesions are more likely to have an organoid pattern with low cellularity, whereas malignant tumors are more likely to have an epithelioid growth pattern and invade the mucosa (Fig. 5; 21). Size and mitotic figure counts show some overlap between benign and malignant tumors. Most benign tumors are $<4.5 \mathrm{~cm}$ and have $<2$ mitoses per 50 HPFs (21).

\section{JEJUNUM AND ILEUM}

Not surprisingly, many of the same prognostic features from the duodenum hold true in the jejunum and ileum. Approximately one half of the GISTs in the jejunum and ileum are malignant. Two 
studies that looked expressly at tumors from the jejunum and ileum found that high cellularity, increased mitoses, large size, and mucosal invasion correlated with an adverse outcome $(22,23)$. One of these studies also found that tumor necrosis, severe nuclear pleomorphism, and a lack of skenoid fibers also correlated with a poor prognosis, whereas an organoid growth pattern favored a better prognosis (23). Both studies used a cut-off of 5 mitoses per 50 HPFs, although one used a size of $5 \mathrm{~cm}$ and the other, a size of $7 \mathrm{~cm}(22,23)$.

Morphologically, stromal tumors from the small bowel (all portions) often have condensations of collagen known as skenoid fibers that typically are not seen in gastric GISTs (Fig. 6). Some older studies have suggested that the presence of skenoid fibers was a marker of neural differentiation indicative of a gastrointestinal autonomic nerve tumor (GANT, also known as plexosarcoma). Some investigators believe that GANTs are a subset of stromal tumors with a poor prognosis that require electron microscopy to differentiate them from other GISTs $(24,25)$. A recent study by Lee and colleagues (26) contradicts this, as these investigators found that GANTs are CD117 positive and behave identically to GISTs. Hence, the idea that all (any) stromal tumors need electron microscopy for their classification finally appears to be dead (or at least dying).

\section{COLON}

GISTs of the colon represent a small percentage of all GISTs, so it is not surprising that less is known about the biologic behavior of these lesions. Tworek and colleagues (27) studied 20 GISTs from the abdominal colon and found that half of these lesions were malignant, often with a highly aggressive clinical course. These investigators found that an infiltrative border within the muscularis propria, $>5$ mitoses per $50 \mathrm{HPFs}$, and invasion of the mucosa by tumor correlated with metastasis and/or death (27). Interestingly, tumor size did not correlate with outcome in this study, as an 18-cm-diameter tumor behaved in a benign fashion, whereas a $2.8-\mathrm{cm}$ diameter tumor metastasized (27). Miettinen and colleagues (28) found that colonic GISTs that were $<5 \mathrm{~cm}$ in diameter, that had $<5$ mitoses per 50 HPFs, or that had skenoid fibers had a better prognosis, whereas those with $>5$ mitoses per 50 HPFs were uniformly fatal. These investigators did not find a relationship between invasion of the muscularis propria and outcome. In another study of colorectal stromal tumors, Moyana and colleagues (29) found that tumor size correlated with outcome, whereas mitotic counts did not. These discordant data illustrate the problems found when studying small numbers of tumors.

\section{ANORECTUM}

GISTs occurring in this location are also rare, and once again, only a handful of studies have looked at these tumors separately. Haque and Dean (30) found that small submucosal lesions that lacked mitoses and pleomorphism behaved in a benign fashion, whereas malignant tumors had mitotic rates of $>5$ per 50 HPFs and were typically present within the deep muscularis propria. Those investigators also noted that these lesions would recur locally, often many years after resection, requiring prolonged follow-up to assess the biologic behavior of these tumors (30). Tworek and colleagues (31) also found that these lesions have a protracted course and that they tend to recur before metastasizing. These investigators found that an infiltrative growth pattern within the muscularis propria and size of $>5 \mathrm{~cm}$ correlated with a poor outcome (31). Miettinen and colleagues (32) found that tumor size of $>5 \mathrm{~cm}$ and $>5$ mitoses per 50 HPFs were associated with a high risk of recurrence or metastasis, whereas tumors sized $<2 \mathrm{~cm}$, with few mitoses, had a good prognosis. The vast majority of these anorectal GISTs were spindle cell tumors, and skenoid fibers were not identified (32).

\section{ESOPHAGUS}

As uncommon as colorectal GISTs are, true esophageal GISTs are even more rare. Although the esophagus gives rise to many true smooth muscle tumors that have confounded the literature by being lumped in with GISTs, a recent study from the Armed Forces Institute of Pathology (done during the "c-Kit Era") found only 17 cases of esophageal GISTs (Fig. 7; 33). More than half of these tumors were malignant, with the majority being $>10 \mathrm{~cm}$ in diameter and having $>5$ mitoses per 50 HPFs (33). Morphologically, esophageal GISTs resemble gastric GISTs, with most showing a cellular spindle cell pattern and some showing epithelioid differentiation.

\section{OMENTUM AND MESENTERY}

Although it seems strange that a tumor of the GI tract that supposedly arises from the ICC could also arise outside of the gut, CD117-positive cells can be found in the omentum, just underneath the mesothelial lining. Reith and colleagues (34) reported on 48 extragastrointestinal stromal tumors and found that $39 \%$ were malignant. These investigators found that high cellularity, high mitotic rate, and necrosis correlated with an adverse outcome (34). Histologically, more than half of the tumors were epithelioid, resembling GISTs of the stomach. Although skenoid fibers were not identified, those investigators did note that the tumors had cystic collections of myxoid stroma and hyalinization 


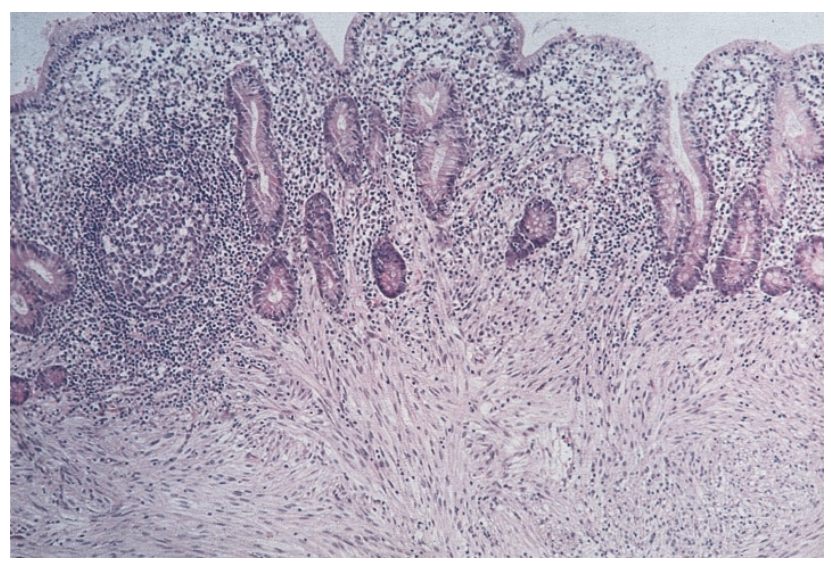

FIGURE 5. Low-power photomicrograph of a malignant small intestinal stromal tumor showing spindle cells invading the mucosa. The presence of tumor cells growing perpendicular to the muscularis mucosae and invading the lamina propria is a sign of malignancy.

around vessels, two findings not typically seen in GISTs from other sites (34). All of these tumors were CD117 positive (34). Miettinen and colleagues (35) found that all of their omental GISTs behaved in a benign fashion, whereas more than half of their mesenteric tumors were malignant. All but one of their tumors were positive for CD117 (35). Many of these extragastrointestinal GISTs seem to resemble gastric stromal tumors histologically, although they behave more like small-intestinal tumors.

\section{DIFFERENTIAL DIAGNOSIS}

Not all mesenchymal lesions of the gut are stromal tumors, and now that effective therapy exists for GISTs, there is added pressure on the surgical pathologist to accurately diagnose these lesions. Other mesenchymal tumors that need to be differentiated from GISTs include inflammatory fibroid polyps, fibromatoses, inflammatory myofibroblastic tumors, solitary fibrous tumors, schwannomas,

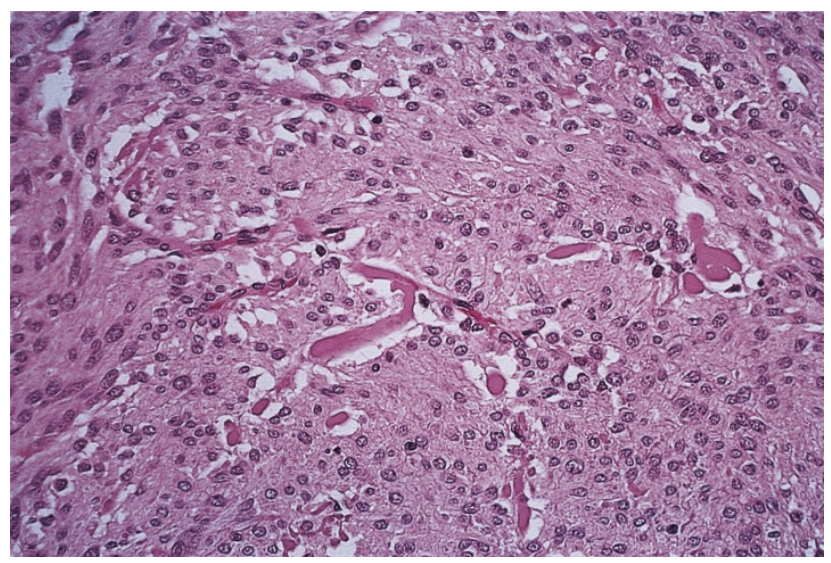

FIGURE 6. High-power photomicrograph of a benign epithelioid small intestinal stromal tumor showing numerous skenoid fibers and low cellularity.

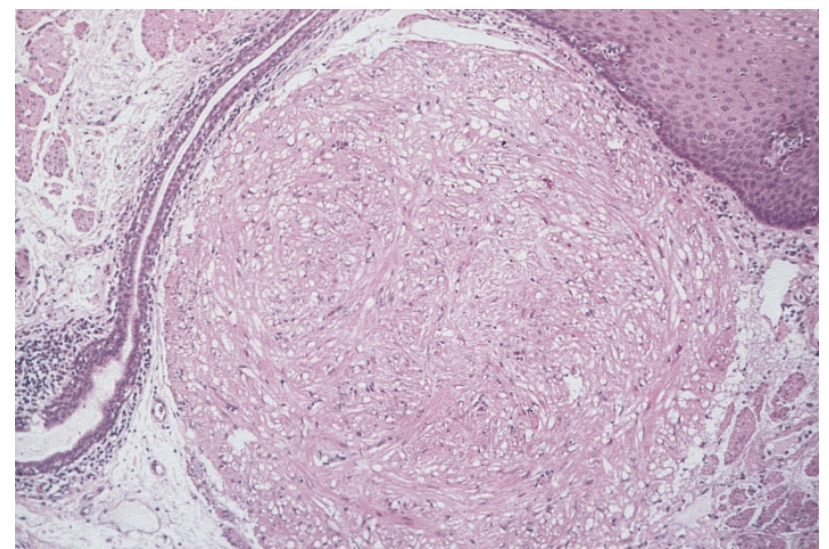

FIGURE 7. Medium-power photomicrograph of a benign incidental leiomyoma of the esophagus. For many years, these small lesions were thought to be stromal tumors, leading to the erroneous impression that most esophageal stromal tumors were benign.

leiomyomas, and leiomyosarcomas. Although immunostains can help one negotiate this minefield of spindle cell lesions, they are not a substitute for experience, as not every CD117-positive tumor is a GIST.

\section{INFLAMMATORY FIBROID POLYPS}

The inflammatory fibroid polyp is a benign lesion most often encountered in the stomach and small intestine. These lesions are typically submucosal and consist of a mixture of small granulation tissue-like vessels, spindle cells, and inflammatory cells (Figs. 8-9). Eosinophils are usually prominent, although plasma cells, lymphocytes, and mast cells are also present. The two most common locations for these tumors are the distal stomach and the terminal ileum (36). Ileal tumors may be transmural with an infiltrative lower border, whereas gastric lesions tend to be confined to the submucosa (36). Rare colonic and esophageal inflammatory fibroid polyps have also been reported. Histologically, the key to the diagnosis is recognizing the presence of

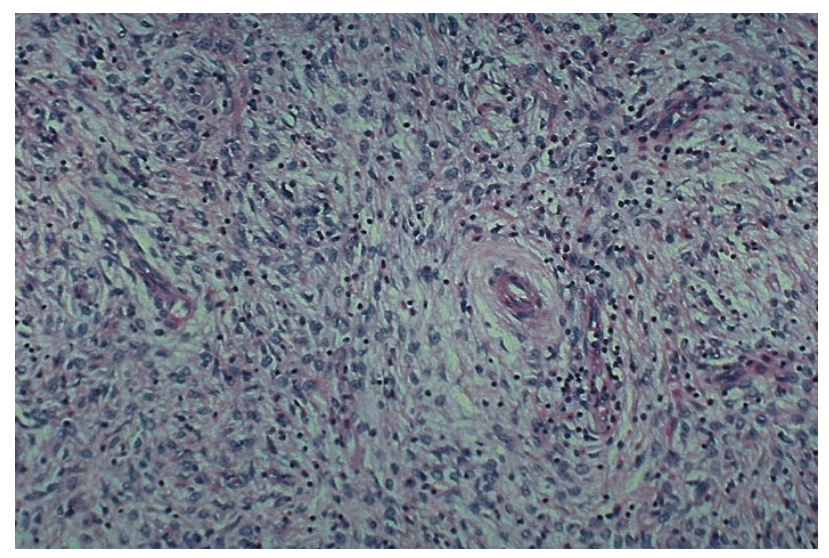

FIGURE 8. Low-power photomicrograph of an inflammatory fibroid polyp showing a granulation tissue-like stroma. 


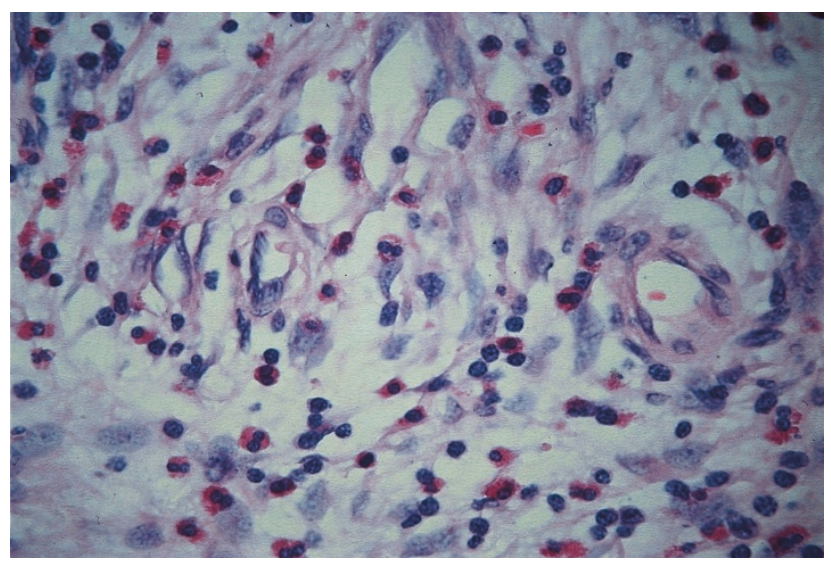

FIGURE 9. High-power photomicrograph of an inflammatory fibroid polyp showing a characteristic eosinophil-rich infiltrate and granulation tissue-like stroma.

many vascular spaces with adjacent eosinophils. Not surprisingly, the majority of these lesions stain positively for CD34, and hence, confusion with GISTs is possible (37). Although the stromal component of these tumors will not stain for CD117, mast cells within the tumor will be positive, providing another possible source of confusion with GISTs.

\section{FIBROMATOSES (DESMOID TUMORS)}

In my consultation practice, the tumors most often confused with GISTs are fibromatoses. These spindle cell tumors can occur in the mesentery or retroperitoneum and grow into the lumen of the gut, mimicking a stromal tumor (38). Histologically, fibromatoses are composed of bland spindled or stellate cells, arranged in parallel with evenly spaced blood vessels and a collagenous background (Figs. 10-11). The amount of collagen is generally greater than that seen in GISTs. Fibromatoses often

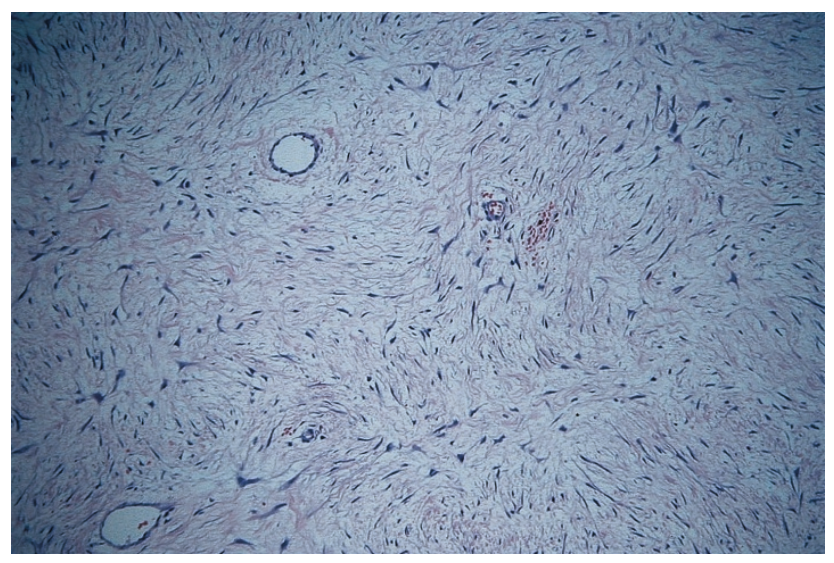

FIGURE 10. Low-power photomicrograph of an abdominal fibromatosis showing bland spindle cells with evenly spaced blood vessels. This lesion has an almost edematous-appearing stroma that contains more collagen than one would expect to see in a GIST.

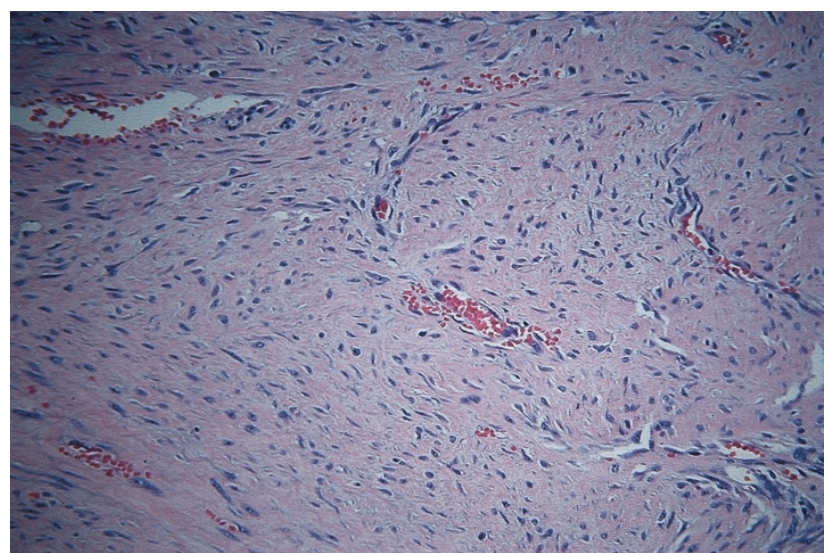

FIGURE 11. Low-power photomicrograph of an abdominal fibromatosis showing a more densely collagenous stroma than the lesion in Figure 10. Again note the evenly spaced blood vessels and the low cellularity of this lesion.

have mitotic activity, but cytologic pleomorphism is generally not seen. Although fibromatoses can be locally aggressive lesions that recur, they do not metastasize and hence should be separated from GISTs (38). Immunostains for CD117 have been reported to be positive in fibromatoses, depending on the antibody used. Yantiss and colleagues (38) reported that $75 \%$ of intra-abdominal fibromatoses stained positive for CD117 using an antibody produced by DAKO. Miettinen (39) confirmed these findings but showed that the antibody made by Santa Cruz Biotechnology did not stain fibromatoses or nodular fasciitis. Interestingly enough, this staining with the Dako antibody has led to some preliminary clinical trials using Gleevec to treat fibromatoses.

\section{INFLAMMATORY MYOFIBROBLASTIC TUMORS}

Inflammatory myofibroblastic tumors (IMTs; also known as inflammatory pseudotumors and inflammatory fibrosarcomas) encompass a family of uncommon mesenchymal lesions that are typified by proliferations of spindle cells admixed with lymphocytes and plasma cells. Although some of these lesions are felt to be benign reactions to infectious processes, others have been shown to be clonal and to rarely behave in a malignant fashion (40). Histologically, these lesions are composed of elongated spindle cells (myofibroblasts) that can mimic GISTs. The presence of an infiltrate of plasma cells should alert the pathologist to the possibility of an IMT, particularly if the patient is young (most GISTs occur in patients $>50 \mathrm{y}$ of age, whereas IMTs are often seen in children). Immunostains for desmin and actin are typically positive, whereas CD117 and CD34 are negative. Sixty percent of IMTs also stain for anaplastic lymphoma kinase (40). 


\section{TRUE SMOOTH MUSCLE TUMORS}

True leiomyomas and leiomyosarcomas do occur in the gut, although they are much less common than GISTs. Most of these lesions arise in the esophagus, although some occur in the colon, rectum, and anus $(28,32,33)$. Leiomyomas resemble the mature smooth muscle of the gut wall, having low cellularity and few mitoses. They stain positively for desmin and actin and negatively for CD117 and CD34 (33). Leiomyomas tend to occur in younger patients than do GISTs (33). Leiomyosarcomas are far less common than leiomyomas and typically have very high mitotic rates. These tumors stain negatively for CD117 and positively for desmin or actin $(32,33)$.

\section{SCHWANNOMAS}

Schwannomas of the gut most often occur in the stomach, although cases have been reported in the colon and esophagus $(41,42)$. These lesions have a characteristic cuff of lymphoid aggregates around their periphery (Fig. 12). The nuclear palisading, Verocay bodies, and hyalinized vessels seen in schwannomas elsewhere in the body are less common or are absent in the gut $(41,42)$. These tumors stain strongly for S-100 (nuclear and cytoplasmic) and are CD117 negative $(41,42)$. Some schwannomas do have focal CD34 positivity (42). It is important to distinguish these lesions from stromal tumors, as schwannomas of the gut are reportedly benign.

\section{SOLITARY FIBROUS TUMORS}

These spindle cell tumors can occasionally occur in the peritoneal cavity and adhere to the bowel. They are described as having a patternless pattern of spindle cells and collagen. These tumors are CD34 positive and hence may be confused with

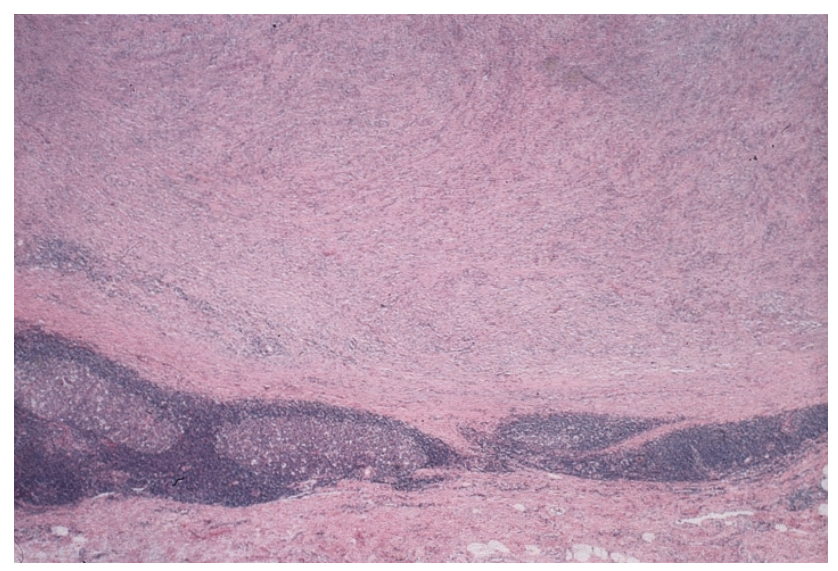

FIGURE 12. Low-power photomicrograph of a gastric schwannoma showing typical cuff of inflammation that sets these lesions apart from stromal tumors.
GISTs. Solitary fibrous tumors do not stain with CD117 (43).

\section{IMMUNOHISTOCHEMISTRY}

Once a quagmire of irreproducible results, the immunohistochemical evaluation of stromal tumors has become much more straightforward since the discovery of CD117. CD117 generally stains GISTs with a diffuse cytoplasmic and/or membranous pattern, although sometimes a perinuclear pattern of staining can be seen (Fig. 13; 44). One should look for positive staining of mast cells in the mucosa as a good internal positive control to ensure that the stain is working properly.

Currently there is some controversy regarding whether a tumor that looks like a GIST on hematoxylin and eosin (H\&E) staining but that is negative for CD117 should be called a GIST. My own bias is that there are rare CD117-negative GISTs but that such a diagnosis should probably only be rendered by an expert. I do think CD117 staining should be done on all stromal tumors, as c-kit positivity is now required for clinical trials using STI571 (Gleevec). However, it is important to recognize that not all CD117-positive tumors are stromal tumors: melanomas, seminomas, acute mylogenous leukemias (including granulocytic sarcomas), angiosarcomas, Ewing's sarcoma, synovial sarcomas, liposarcomas, malignant fibrous histiocytomas, dermatofibrosarcoma protuberans, hemangiopericytomas, and fibromatoses (depending on the antibody used) all have been reported to stain with CD117 (38, 39, 43-46). It goes without saying that a positive CD117 stain needs to be interpreted within the context of the H\&E findings.

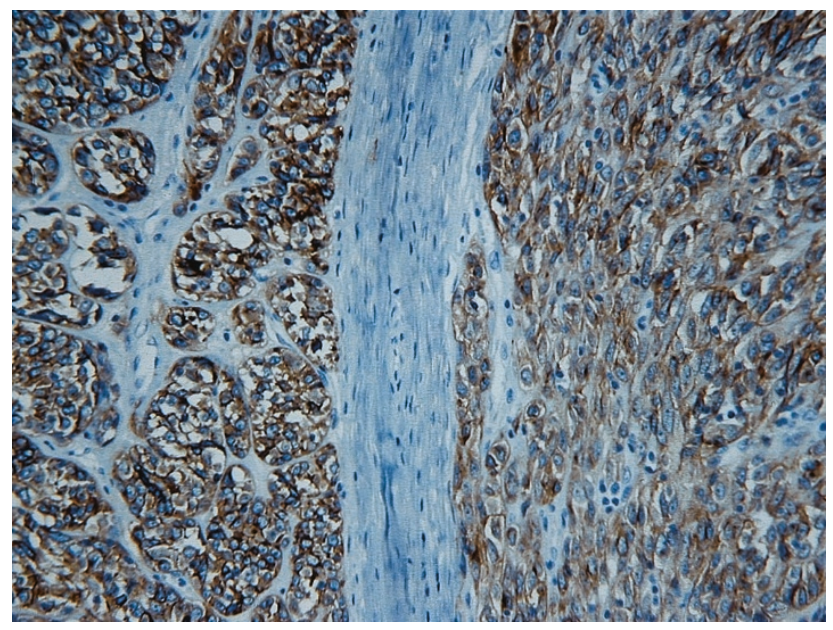

FIGURE 13. Medium-power photomicrograph of a stromal tumor showing strong positive cytoplasmic and membranous staining for CD117. This staining pattern is typical of most GISTs. 


\section{MANAGEMENT ISSUES}

In today's world of doing more with less, pathologists are being asked to make diagnoses on the basis of less and less tissue. Making the diagnosis of a GIST on a needle or mucosal biopsy is very difficult, to say the least. Unless the tumor is extremely high grade, it is unlikely that any prognostic information can be given from such a small sample. Many malignant GISTs have areas that appear benign, such that sampling can be a huge problem. It is also conceivable that the area biopsied could be CD117 negative whereas other parts of the tumor are positive, leading to a completely erroneous diagnosis.

The use of frozen sections in the diagnosis of GISTs is another potential minefield for the surgical pathologist. As these lesions are sometimes discovered by surprise during an exploratory laparotomy, the pathologist may be faced with making a diagnosis on frozen section. Again, unless the lesion is obviously high grade, I would avoid calling the lesion benign or malignant on frozen section.

The cost-effective use of immunoperoxidase stains is another management issue for the surgical pathologist. Because of the effectiveness of Gleevec, I believe that all stromal tumors should be stained with CD117, much like all breast cancers are stained for estrogen and progesterone receptors and HER-2/neu. In cases in which I am not sure whether the lesion is a stromal tumor based on the H\&E-stained sections, I usually order CD34, S-100, and smooth muscle actin (in addition to CD117). I do not think an extensive battery of immunostains is necessary in the vast majority of cases.

How one signs out stromal tumors is a matter of individual preference; however, there are certain parameters that I believe should be mentioned in the surgical pathology report. At a bare minimum, the size, mitotic rate, and status of the surgical margins should be mentioned. I also include the presence or absence of mucosal invasion and the cellularity of the lesion. I then summarize these findings by stating that the tumor is one of the following: histologically benign, malignant, or of uncertain malignant potential. Some experts feel that all stromal tumors are potentially malignant and hence that tumors should be classified as low risk and high risk, whereas others prefer not to mention prognosis at all.

\section{THE FUTURE}

The debate on how best to predict the biologic behavior of stromal tumors will undoubtedly persist for some time, making it difficult to give absolute recommendations on how to evaluate these lesions. Although some studies have found value in ancillary studies such as ploidy analysis, the use of proliferation markers, and the detection of C-kit mutations, to date we still have no consensus on the ultimate utility of these techniques, as none are accurate enough to predict tumor behavior in a given patient (47-52).

Although we have just scratched the surface of the molecular genetic alterations involved with stromal tumors, significant progress has been made. The identification of functional c-kit mutations in the development of these tumors and the production of a designer drug that successfully treats these lesions by interfering with this pathway is an exciting start to the new millennium. The majority of mutations in the kit gene in GISTs have involved the juxtamembrane domain (exon 11; 51, 52). These mutations lead to ligand-independent activation of the tyrosine kinase (autophosphorylation) and have been associated with a poor prognosis $(51,52)$. To date, however, the percentage of GISTs with exon 11 mutations has ranged from only 21 to $57 \%$, indicating that other genetic alterations are probably involved (51-53). Mutations in other exons (exons 3 and 9) of c-kit have also been found, but these seem to be present in a small minority of tumors (54). Other genetic alterations in GISTs, which appear to be independent of c-kit gene mutations, include $\mathrm{LOH}$ at $1 \mathrm{p}, 14 \mathrm{q}$, and $22 \mathrm{q}$ (55). The $\mathrm{LOH}$ at $22 \mathrm{q}$ is very interesting because it corresponds to the locus of the NF2 gene (55).

The application of microarray technology to the study of GISTs will undoubtedly uncover more genetic hot spots involved in the pathogenesis of these tumors. Until then, c-kit and its inhibitor STI571 will remain in the spotlight. Despite all of the above referenced technologic breakthroughs, there is still no substitute for the intelligent analysis of "old-fashioned" H\&E-stained sections.

Acknowledgment: The author thanks Henry D. Appelman, M.D., for the use of his photomicrographs.

\section{REFERENCES}

1. Martin JF, Bazin P, Feroldi J, Cabanne F. Tumeurs myoides intra-murales de l'estomac; consideration microscopiques a propos de 6 cas. Ann Anat Pathol 1960;5:484-97.

2. Stout AP. Bizarre smooth muscle tumors of the stomach. Cancer 1962;15:400-9.

3. Mazur MT, Clark HB. Gastric stromal tumors: reappraisal of histogenesis. Am J Surg Pathol 1983;7:507-19.

4. Miettinen M, Virolainen M, Sarlomo-Rikala M. Gastrointestinal stromal tumors-value of CD34 antigen in their identification and separation from true leiomyomas and schwannomas. Am J Surg Pathol 1995;19:207-16.

5. Monihan JM, Carr NJ, Sobin LH. CD34 immunoexpression in stromal tumours of the gastrointestinal tract and in mesenteric fibromatoses. Histopathology 1994;25:469-73.

6. Vliagoftis H, Worobec AS, Metcalfe DD. The protooncogene c-kit and c-kit ligand in human disease. J Allergy Clin Immunol 1997;100:435-40. 
7. Hirota S, Isozaki K, Moriyama Y, Hashimoto K, Nishida T, Ishiguro S, et al. Gain-of-function mutations of c-kit in human gastrointestinal stromal tumors. Science 1998;279:57780 .

8. Beghini A, Tibiletti MG, Roversi G, Chiaravalli AM, Serio G, Capella C, et al. Germline mutation in the juxtamembrane domain of the kit gene in a family with gastrointestinal stromal tumors and urticaria pigmentosa. Cancer 2001;92: 657-62.

9. Kindblom LG, Remotti HE, Aldenborg F, Meis-Kindblom JM. Gastrointestinal pacemaker cell tumor (GIPACT): gastrointestinal stromal tumors show phenotypic characteristics of the interstitial cells of Cajal. Am J Pathol 1998;152:1259-69.

10. Druker BJ, Tamura S, Buchdunger E, Ohno S, Segal GM, Fanning S, et al. Effects of a selective inhibitor of the Abl tyrosine kinase on the growth of Bcr-Abl positive cells. Nat Med 1996;2:561-6.

11. Drucker BJ, Talpaz M, Resta DJ, Peng B, Buchdunger E, Ford JM, et al. Efficacy and safety of a specific inhibitor of the BCR-ABL tyrosine kinase in chronic myeloid leukemia. N Engl J Med 2001;344:1031-7.

12. Joensuu H, Roberts PJ, Sarlomo-Rikala M, Andersson LC, Tervahartiala $\mathrm{P}$, Tuveson $\mathrm{D}$, et al. Effect of the tyrosine kinase inhibitor STI571 in a patient with a metastatic gastrointestinal stromal tumor. N Engl J Med 2001;344:1052-6.

13. Emory TS, Sobin LH, Lukes L, Lee DH, O'Leary TJ. Prognosis of gastrointestinal smooth-muscle (stromal) tumors: dependence on anatomic site. Am J Surg Pathol 1999;23:82-7.

14. Miettinen M, Sarlomo-Rikala M, Lasota J. Gastrointestinal stromal tumors: recent advances in understanding of their biology. Hum Pathol 1999;30:1213-20.

15. Berman J, O'Leary TJ. Gastrointestinal stromal tumor workshop. Hum Pathol 2001;32:578-82.

16. Appelman HD. Smooth muscle tumors of the gastrointestinal tract. What we know now that Stout didn't know. Am J Surg Pathol 1986;1(10 Suppl):83-99.

17. Eckhauser FE, Yahanda AM, Greenson JK. Gastric smooth muscle tumors. In: Cameron JL, editor. Current surgical therapy. 6th ed. Philadelphia, PA: Mosby Electronic Production; 1998. p. 114-7.

18. Appelman HD, Helwig EB. Cellular leiomyomas of the stomach in 49 patients. Arch Pathol Lab Med 1977;101:373-7.

19. Appelman HD, Helwig EB. Gastric epithelioid leiomyoma and leiomyosarcoma (leiomyoblastoma). Cancer 1976;38: 708-28.

20. Carney JA, Sheps SG, Go VL, Gordon H. The triad of gastric leiomyosarcoma, functioning extra-adrenal paraganglioma and pulmonary chondroma. N Engl J Med 1977;296:1517-8.

21. Goldblum JR, Appelman HD. Stromal tumors of the duodenum: a histologic and immunohistochemical study of 20 cases. Am J Surg Pathol 1995;19:71-80.

22. Tworek JA, Appelman HD, Singleton TP, Greenson JK. Stromal tumors of the jejunum and ileum. Mod Pathol 1997;10: 200-9.

23. Brainard JA, Goldblum JR. Stromal tumors of the jejunum and ileum: a clinicopathologic study of 39 cases. Am J Surg Pathol 1997;21:407-16.

24. Lauwers GY, Erlandson RA, Casper ES, Brennan MF, Woodruff JM. Gastrointestinal autonomic nerve tumors: a clinicopathologic, immunohistochemical and ultrastructural study of 12 cases. Am J Surg Pathol 1993;17:887-97.

25. Rosai J. Ackerman's surgical pathology. 8th ed. St. Louis, MO: Mosby; 1996. p. 645-7.

26. Lee JR, Joshi V, Griffen JW Jr, Lasota J, Miettinen M. Gastrointestinal autonomic nerve tumor: immunohistochemical and molecular identity with gastrointestinal stromal tumor. Am J Surg Pathol 2001;25:979-87.
27. Tworek JA, Goldblum JR, Weiss SW, Greenson JK, Appelman HD. Stromal tumors of the abdominal colon. Am J Surg Pathol 1999;23:937-45.

28. Miettinen M, Sarlomo-Rikala M, Sobin LH, Lasota J. Gastrointestinal stromal tumors and leiomyosarcomas in the colon: a clinicopathologic, immunohistochemical, and molecular genetic study of 44 cases. Am J Surg Pathol 2000;24: $1339-52$.

29. Moyana TN, Friesen R, Tan LK. Colorectal smooth-muscle tumors: a pathologic study with immunohistochemistry and histomorphometry. Arch Pathol Lab Med 1991;115:1016-21.

30. Haque S, Dean PJ. Stromal neoplasms of the rectum and anal canal. Hum Pathol 1992;23:762-7.

31. Tworek JA, Goldblum JR, Weiss SW, Greenson JK, Appelman HD. Stromal tumors of the anorectum. Am J Surg Pathol 1999;23:946-54.

32. Miettinen M, Furlong M, Sarlomo-Rikala M, Burke A, Sobin LH, Lasota J. Gastrointestinal stromal tumors, intramural leiomyomas, and leiomyosarcomas in the rectum and anus: a clinicopathologic, immunohistochemical, and molecular genetic study of 144 cases. Am J Surg Pathol 2001;25:112133.

33. Miettinen M, Sarlomo-Rikala M, Sobin LH, Lasota J. Esophageal stromal tumors: a clinicopathologic, immunohistochemical, and molecular genetic study of 17 cases and comparison with esophageal leiomyomas and leiomyosarcomas. Am J Surg Pathol 2000;24:211-22.

34. Reith JD, Goldblum JR, Lyles RH, Weiss SW. Extragastrointestinal (soft tissue) stromal tumors: an analysis of 48 cases with emphasis on histologic predictors of outcome. Mod Pathol 2000;13:577-85.

35. Miettinen M, Monihan JM, Sarlomo-Rikala M, Kovatich AJ, Carr NJ, Emory TS, et al. Gastrointestinal stromal tumors/ smooth muscle tumors (GISTs) primary in the omentum and mesentery: clinicopathologic and immunohistochemical study of 26 cases. Am J Surg Pathol 1999;23:1109-18.

36. Appelman HD. In: Ming SC, Goldman H, editors. Pathology of the gastrointestinal tract. Philadelphia, PA: Saunders; 1992. p. 310-50.

37. Kim MK, Higgins J, Cho EY, Ko YH, Oh YL. Expression of CD34, bcl-2, and kit in inflammatory fibroid polyps of the gastrointestinal tract. Appl Immunohistochem Mol Morphol 2000;8:147-53.

38. Yantiss RK, Spiro IJ, Compton CC, Rosenberg AE. Gastrointestinal stromal tumor versus intra-abdominal fibromatosis of the bowel wall: a clinically important differential diagnosis. Am J Surg Pathol 2000;24:947-57.

39. Miettinen M. Are desmoid tumors KIT positive? Am J Surg Pathol 2001;25:549-50.

40. Cook JR, Dehner LP, Collins MH, Ma Z, Morris SW, Coffin $\mathrm{CM}$, et al. Anaplastic lymphoma kinase (ALK) expression in the inflammatory myofibroblastic tumor. Am J Surg Pathol 2001;25:1364-71.

41. Prevot S, Bienvenu L, Vaillant JC, de Saint-Maur PP. Benign schwannoma of the digestive tract: a clinicopathologic and immunohistochemical study of five cases, including a case of esophageal tumor. Am J Surg Pathol 1999;23:431-6.

42. Miettinen M, Shekitka KM, Sobin LH. Schwannomas in the colon and rectum: a clinicopathologic and immunohistochemical study of 20 cases. Am J Surg Pathol 2001;25:846-55.

43. Sarlomo-Rikala M, Kovatich AJ, Barusevicius A, Miettinen M. CD117: a sensitive marker for gastrointestinal stromal tumors that is more specific than CD34. Mod Pathol 1998;11: 728-34.

44. Miettinen M, Sobin LH, Sarlomo-Rikala M. Immunohistochemical spectrum of GISTs at different sites and their differential diagnosis with a reference to CD117 (KIT). Mod Pathol 2000;13:1134-42. 
45. Strickland L, Letson GD, Muro-Cacho CA. Gastrointestinal stromal tumors. Cancer Control 2001;8:252-61.

46. Tamborini E, Papini D, Mezzelani A, Riva C, Azzarelli A, Sozzi G, et al. c-KIT and c-KIT ligand (SCF) in synovial sarcoma (SS): an mRNA expression analysis in 23 cases. $\mathrm{Br}$ J Cancer 2001;85:405-11.

47. Lerma E, Lee SJ, Tugues D, Oliva E, Gich I, Prat J. Ploidy of 36 stromal tumors of the gastrointestinal tract. A comparative study with flow cytometry and image analysis. Anal Quant Cytol Histol 1994;16:435-40.

48. Lerma E, Oliva E, Tugues D, Prat J. Stromal tumours of the gastrointestinal tract: a clinicopathological and ploidy analysis of 33 cases. Virchows Archiv 1994;424:19-24.

49. Cooper PN, Quirke P, Hardy GJ, Dixon MF. A flow cytometric, clinical, and histological study of stromal neoplasms of the gastrointestinal tract. Am J Surg Pathol 1992;16:163-70.

50. Yu CC, Fletcher CD, Newman PL, Goodlad JR, Burton JC, Levison DA. A comparison of proliferating cell nuclear antigen (PCNA) immunostaining, nucleolar organizer region (AgNOR) staining, and histological grading in gastrointestinal stromal tumours. J Pathol 1992;166:147-52.
51. Taniguchi M, Nishida T, Hirota S, Isozaki K, Ito T, Nomura T, et al. Effect of c-kit mutation on prognosis of gastrointestinal stromal tumors. Cancer Res 1999;59:4297-300.

52. Lasota J, Jasinski M, Sarlomo-Rikala M, Miettinen M. Mutations in exon 11 of c-Kit occur preferentially in malignant versus benign gastrointestinal stromal tumors and do not occur in leiomyomas or leiomyosarcomas. Am J Pathol 1999; 154:53-60.

53. Moskaluk CA, Tian Q, Marshall CR, Rumpel CA, Franquemont DW, Frierson HF Jr. Mutations of c-kit JM domain are found in a minority of human gastrointestinal stromal tumors. Oncogene 1999;18:1897-902.

54. Lasota J, Wozniak A, Sarlomo-Rikala M, Rys J, Kordek R, Nassar A, et al. Mutations in exons 9 and 13 of KIT gene are rare events in gastrointestinal stromal tumors. A study of 200 cases. Am J Pathol 2000;157:1091-5.

55. Fukasawa T, Chong JM, Sakurai S, Koshiishi N, Ikeno R, Tanaka A, et al. Allelic loss of 14q and 22q, NF2 mutation, and genetic instability occur independently of c-kit mutation in gastrointestinal stromal tumor. Jpn J Cancer Res 2000;91:1241-9. 